\title{
A Review on Advances in Automated Plant Disease Detection
}

\author{
Radhika Bhagwat $^{1,2, *}$, Yogesh Dandawate ${ }^{3}$ \\ ${ }^{1}$ Department of Technology, Savitribai Phule Pune University, Pune, India \\ ${ }^{2}$ Department of Information Technology, Cummins College of Engineering for Women, Pune, India \\ ${ }^{3}$ Electronics and Telecommunication Engineering, Vishwakarma Institute of Information Technology, Pune, India \\ Received 09 August 2021; received in revised form 25 August 2021; accepted 26 August 2021 \\ DOI: https://doi.org/10.46604/ijeti.2021.8244
}

\begin{abstract}
Plant diseases cause major yield and economic losses. To detect plant disease at early stages, selecting appropriate techniques is imperative as it affects the cost, diagnosis time, and accuracy. This research gives a comprehensive review of various plant disease detection methods based on the images used and processing algorithms applied. It systematically analyzes various traditional machine learning and deep learning algorithms used for processing visible and spectral range images, and comparatively evaluates the work done in literature in terms of datasets used, various image processing techniques employed, models utilized, and efficiency achieved. The study discusses the benefits and restrictions of each method along with the challenges to be addressed for rapid and accurate plant disease detection. Results show that for plant disease detection, deep learning outperforms traditional machine learning algorithms while visible range images are more widely used compared to spectral images.
\end{abstract}

Keywords: plant disease detection, visible range image, spectral image, traditional machine learning, deep learning

\section{Introduction}

Agriculture is one of the dominant economic sectors for developing countries as it provides main livelihood sources for rural population. In some developing countries, agriculture accounts for more than $25 \%$ of gross domestic product (GDP) [1]. The demand of a constantly rising population can be met by an increase in the yield. However, the losses due to crop diseases and pests significantly influence the contribution made by agricultural products. Additionally, dynamic weather conditions also spread diseases rapidly, thus aggravating the problem of food security. Therefore, the responsibility of controlling crop losses in the initial stages is important, not only for the growing economy and food supplement for animals and humans, but also to maintain the ecosystem balance. In response to these challenges, there is increased attention for adapting precision agriculture (PA) practices to achieve a sustained increase in efficiency and yields.

Various methods are being used for early plant disease detection. The traditional method of diagnosis by an agronomist is based on a visible inspection of the affected plants. However, this method is labor-intensive, costly, and less accurate. Also, many producers from rural extensions do not have access to this technical advice, resulting in a high risk of yield losses due to crop diseases. The laboratory based tests have limitations due to their complex methods and time requirements. Because of the drawbacks in laboratory based methods, non-invasive methods have gained attention during the last few decades. A lot of work is being done in this area and the aim is to develop a mechanism that is automated, fast, and accurate to subdue the constraints of traditional methods. One of the most popular methods to meet the above-mentioned requirements is the use of various image processing techniques. Numerous cameras with highly sensitive sensors have been developed in recent years and are being

* Corresponding author. E-mail address: radbhag@gmail.com 
used to obtain the information from crops. The types of imaging systems involve visible light, spectral, thermal, fluorescence imaging, etc. The images captured by suitable imaging tools are processed using a variety of image processing approaches, and are then utilized to train/test machine learning algorithms. All the earlier work used classical machine learning techniques to identify diseases. However, the automated systems using classical machine learning algorithms faced limitations in terms of small datasets and hand crafted feature extraction methods, leading to limited performance and limited crop and disease scope.

With the revolution in areas like computer vision and graphics processing unit (GPU), deep learning is considered to be a capable tool to enhance automated techniques to achieve higher performance, wide crop and disease range, and real-time disease identification. In recent years, researchers are working on the development of deep learning architectures for automatic feature extraction and disease classification. This study does a thorough review of several classical machine learning and deep learning architectures using RGB and spectral imaging techniques (i.e., hyperspectral and multispectral techniques) for plant disease recognition. It also discusses the merits and limitations of each method, and further describes the challenges that need to be addressed for the fast, accurate, and real-time plant disease detection. The various techniques for plant disease detection discussed in this study are presented in Fig. 1.

The arrangement of the rest of the study is as follows. Section 2 reviews the related work done towards the use of image processing algorithms for pest and disease identification. Section 3 deals with the discussion about the merits and limitations of the methods, and also takes note of the challenges to be addressed for a robust crop disease diagnosis system. Finally, section 4 presents the conclusions.

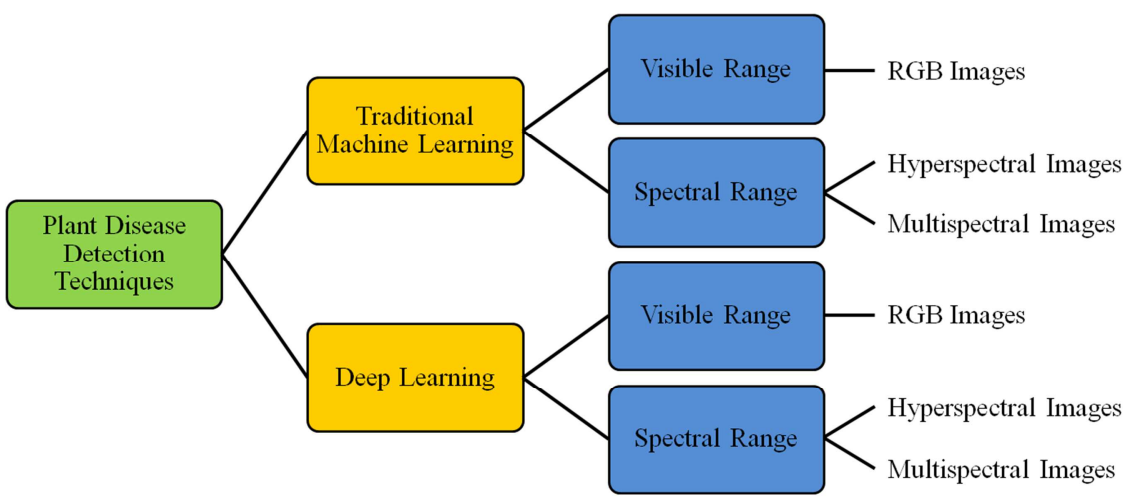

Fig. 1 Various techniques discussed for plant disease detection

\section{Related Work}

To detect plant diseases in early stages, researchers have been working on numerous techniques for many decades by using invasive and non-invasive methods at various stages, and have found that the techniques are successful at different levels. However, recent developments in agricultural technology demand an automated non-invasive method for plant disease diagnosis. Due to the improved potential of numerous cameras with highly sensitive sensors to capture crop details, various image processing methods are used in the automatic plant disease recognition task to have a system that is accurate and takes less effort and time. The symptoms of a disease can appear on any part of the plant, e.g., leaf, root, fruit, flower, and stem. Most of the work in literature has been done majorly on leaf images although there are methods that take into account images of stems [2], fruits [3], and also the entire plant [4].

This section provides a thorough review of traditional machine learning and deep learning algorithms for disease detection. Section 2.1. reviews the classical machine learning techniques based on RGB and spectral images used in crop disease detection, while section 2.2. focuses on the review of the work regarding deep learning architectures applied on visible light images and spectral images. 


\subsection{Traditional machine learning for plant disease detection}

Machine learning methods are utilized to find important fundamental patterns within complex data. Early work in the area of disease detection used traditional machine learning methods for the classification of images. The generic steps used for plant disease recognition and classification with traditional machine learning algorithms are shown in Fig. 2.

The first step is to create a database which may involve capturing the images using a suitable imaging system or using a publicly available dataset. Image preprocessing is a vital start required to enhance image characteristics and to reduce the time required for processing in further steps. Some of the popular pre-processing steps involve image resizing, noise removal, contrast enhancement, conversion of color space, etc. Image segmentation is done to get the target region from the entire image. Few popularly used segmentation techniques are thresholding, K-means clustering, etc. After segmentation is done, relevant features such as shape, size, texture, and color are extracted from the segmented images. With the help of these extracted feature vectors, the machine learning algorithms are trained to label the images into given categories. Numerous classifiers, e.g., support vector machine (SVM), naive Bayes, artificial neural network (ANN), etc., are used for classifying images. The use of test data is done on the trained model to categorize the new data into one of the distinct classes. The potential of the model is assessed using various evaluation metrics such as accuracy, precision, F1-score, and area under curve (AUC).

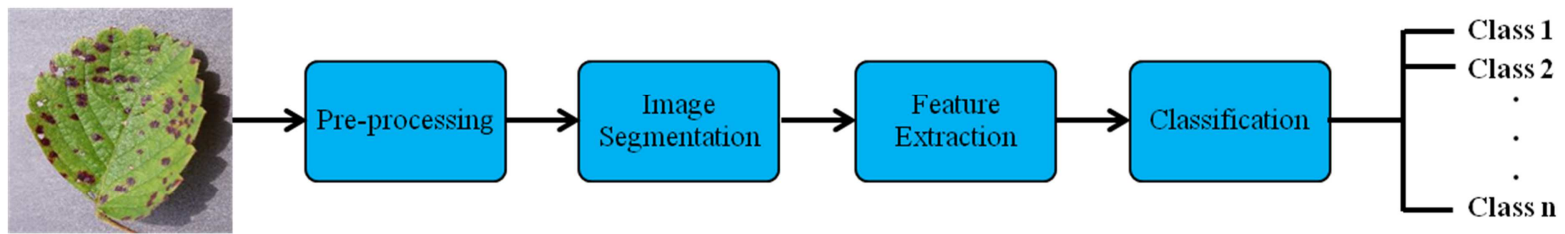

Input

Fig. 2 General steps in traditional machine learning

\subsubsection{Traditional machine learning with $R G B$ images}

Considerable work has been done in this area, with each disease detection system recommending a distinct way for classification, segmentation, etc. Ali et al. [5] presented a method for the identification and classification of citrus diseases based upon visual symptoms. The distance between colors was used for segmenting the infected area in images. Based on the color histogram and texture features, citrus greening, anthracnose, downy, and healthy citrus leaves were labeled. Local binary pattern (LBP) and color features were used and the method was tested on various classifiers like SVM, K-nearest neighbor $(\mathrm{KNN})$, boosted tree, and bagged tree. The authors applied disease level along with image level classification and reported good discrimination with color features for disease level classification. The overall accuracy of $99 \%$ and sensitivity of $99.7 \%$ were reported. The experiment with the combination of color and texture features gave an under-performance compared to individual features. The database consisted of only 199 images (including 100 images for healthy citrus and 33 images each for three citrus diseases). Islam et al. [6] proposed classifying potato diseases using images taken from plant village dataset [7]. The authors segmented the images by generating the masks based on La*b* color model. Using 10 texture and color features and multiclass SVM, the work reported an accuracy of 95\%. However, the study used only 300 images for experimentation while plant village dataset has a large number of potato leaf images. In these studies [5-6], the performance would have been improved with a larger dataset.

Zhang et al. [8] presented a technique for detecting citrus canker based upon global features and zone-based local features from field captured leaf images. The authors utilized an improved AdaBoost algorithm for segmenting the lesions from the background, and then combined the color and the distribution of the local texture for generating a canker lesion descriptor. A two-step hierarchical structure to identify citrus canker lesions was developed that obtained the classification accuracy similar to human experts. 
Sharif et al. [3] proposed an algorithm for the detection of lesions on citrus fruits and leaves using three different datasets. The use of an optimized weighted segmentation technique was done on the pre-processed images. A codebook was generated from the color, texture, and geometric features, and the best features were selected using a hybrid feature selection technique. The classification was done using a multiclass SVM that got an average accuracy of 92.435\%. Hassanien et al. [9] used an enhanced moth-flame approach based upon rough sets to detect the diseases (i.e., powdery mildew and early blight) in tomato leaves. A feature selection technique was presented and the SVM algorithm was used to classify the diseased tomato leaves. The study compared the performance of the proposed moth flame optimization technique with particle swarm optimization (PSO) and genetic algorithm (GA). The proposed feature selection method showed an improvement of $6 \%$ in the classification accuracy. These studies [3,9] demonstrated that with correct feature selection, the classifier performance can be enhanced.

Singh et al. [10] proposed image segmentation techniques based on GA for automatic leaf disease detection and classification for five different classes: bacterial disease on rose, bacterial disease on bean leaves, lemon leaves with sunburn, early scorch on banana leaf, and beans with fungal disease. A color co-occurrence matrix was utilized to get four texture features. The classification was done with the minimum distance criterion (MDC) and SVM. The authors reported a classification accuracy of $86.54 \%, 93.63 \%$, and $95.71 \%$ for MDC with K-means, MDC with the proposed GA, and SVM with the proposed GA, respectively. This study shows that the learning algorithms can be used for lesion segmentation.

The identification of multiple plant diseases in uncontrolled conditions using digital image processing was proposed in the work of Barbedo et al. [11]. They used a dataset of 12 plant species with 82 different disorders. Background removal was done using the guided active contour (GAC) method. Symptom segmentation used a binary mask and two ratios to indicate the variation of each pixel from green. The color transformation was used to get different characteristics suitable for the identification of symptoms. The training was done using color histograms to capture the general behavior of the disease, and pairwise classification was done using a reference histogram. A confusion matrix was used to show the results. The study also discussed challenges, e.g., the correspondence between diseases and the difference in the conditions of image captured that were responsible for the error rate. Omrani et al. [12] detected three different apple diseases (Alternaria, black spot, and leaf miner pest) using image processing methods. The images were captured in a laboratory condition and segmented using K-means clustering. Wavelet and gray level co-occurrence matrix were used to get textural features and color features extracted using La*b* color space. The authors used SVM with a radial basis function (RBF), a polynomial function (poly), and ANN classifiers to identify diseases in apple leaves. The algorithm was tested on the images taken with proper lighting conditions and black background.

Phadikar et al. [13] used Fermi energy based segmentation methods to classify four rice diseases: leaf brown spot, leaf blast, sheath rot, and bacterial blight. Color, shape, and position of the infection were used as features and the rough set theory was utilized for selecting prominent features. A rule based classifier was used for classifying the rice diseases to get $92.29 \%$ accuracy. The authors also compared their proposed method with the existing feature selection and classification techniques and with the benchmark UCI dataset, and attained an accuracy of $80.39 \%$ and $94.21 \%$ respectively. The classifier complexity can be reduced by selecting principle features to lessen the information loss. However, the compromise between feature dimension and loss of information is always crucial.

Barbedo [2] presented a survey on different methods for detecting and classifying plant diseases along with measuring severity. The author used digital images of leaves and stems in a visible spectrum. Barbedo [14] presented a technique to identify and quantify leaf disease symptoms automatically using image processing techniques. The author used simple morphological operations and a* channel in $\mathrm{La}^{*} \mathrm{~b}^{*}$ space for detecting diseases, and achieved an overall classification accuracy of $96 \%$. However, the algorithm required the image to be captured under white or black background. 
Camargo et al. [15] described a method to detect visual symptoms on plant leaves. After the color transformation of RGB images, segmentation was done using intensity distribution in the histogram and local maxima for thresholding. The automatic segmentation method was compared with a manual segmentation method to test the accuracy of the algorithm. The authors extend their work by using the identified target regions [16]. Relevant features were extracted from the target regions and were classified using SVM. The authors reported that texture features can give the best discrimination when the images do not have clear color and shape. These studies demonstrated the importance of using appropriate hand crafted features for improving the classifier performance.

Johannes et al. [17] proposed a method based upon recognition of a candidate hot-spot and statistical reasoning for identifying three wheat diseases (i.e., Septoria, rust, and tan spot) in real field conditions. The study reported two alternatives for segmentation: i) using manually generated masks; ii) using simple linear iterative clustering (SLIC) and visual features. The disease candidate regions were extracted and analyzed using local descriptors. The study reported an AUC greater than 0.8 using a meta classifier. The authors reported the algorithm to work on different crops and diseases, and deployed it on a smartphone application. The authors used color constancy algorithm to handle the illumination changes that occur in field conditions, proving that color constancy can be used to normalize the illumination variations.

Table 1 gives a summary of the distinct research work done using classical machine learning algorithms on RGB images. It summarizes the crops used, various pre-processing and segmentation methods applied, relevant extracted features along with the classifiers, and the performance metric used in the studies.

Table 1 Comparative study of classical machine learning algorithm on RGB images

\begin{tabular}{|c|c|c|c|c|c|c|c|c|}
\hline Ref. & Crop & $\begin{array}{c}\text { Dataset } \\
\text { (no. of images) }\end{array}$ & $\begin{array}{l}\text { Plant part } \\
\text { used }\end{array}$ & $\begin{array}{l}\text { Pre-processing } \\
\text { method }\end{array}$ & Segmentation method & Extracted features & Classifier & Evaluation metric \\
\hline$[3]$ & Citrus & $\begin{array}{c}\text { Own-580, } \\
\text { citrus disease image } \\
\text { gallery-1000, and } \\
\text { combined-5632 }\end{array}$ & $\begin{array}{l}\text { Fruits } \\
\text { and } \\
\text { leaves }\end{array}$ & $\begin{array}{l}\text { Hybrid contrast } \\
\text { stretching } \\
\text { technique }\end{array}$ & $\begin{array}{l}\text { Enhanced weighted } \\
\text { segmentation }\end{array}$ & $\begin{array}{l}\text { Color, textural, and } \\
\text { geometric features }\end{array}$ & Multiclass SVM & $\begin{array}{c}\text { Average accuracy }= \\
92.435 \%\end{array}$ \\
\hline$[5]$ & Citrus & 199 & Leaves & $\begin{array}{c}\text { Image } \\
\text { enhancement } \\
\text { and color } \\
\text { transformation }\end{array}$ & $\begin{array}{l}\text { Color difference based } \\
\text { algorithm }\end{array}$ & $\begin{array}{l}\text { Color histogram, } \\
\text { LBP }\end{array}$ & $\begin{array}{c}\mathrm{KNN}, \mathrm{SVM} \text {, boosted tree, and } \\
\text { bagged tree }\end{array}$ & $\begin{array}{c}\text { Sensitivity }=99.7 \% \\
\text { Accuracy }=99 \% \\
\text { AUC }=1.0\end{array}$ \\
\hline [9] & Tomato & 200 & Leaves & $\begin{array}{l}\text { Noise removal } \\
\text { and image } \\
\text { resizing }\end{array}$ & $\begin{array}{l}\text { Gaussian mixture based } \\
\text { background/foreground } \\
\text { segmentation }\end{array}$ & $\begin{array}{c}\text { Textural patterns } \\
\text { with moth flame } \\
\text { optimization based } \\
\text { rough set }\end{array}$ & SVM & $\begin{array}{l}\text { Accuracy }=91.5 \\
\text { Precision }=91.5 \\
\text { Recall }=91.5 \%\end{array}$ \\
\hline [10] & $\begin{array}{l}\text { Rose, } \\
\text { bean, } \\
\text { lemon, } \\
\text { and } \\
\text { banana }\end{array}$ & $\begin{array}{l}25 \text { images for } \\
\text { each class }\end{array}$ & Leaves & $\begin{array}{l}\text { Clipping, } \\
\text { image } \\
\text { smoothing, and } \\
\text { contrast } \\
\text { enhancement }\end{array}$ & GA & $\begin{array}{l}\text { Color co-occurrence } \\
\text { matrix: local } \\
\text { homogeneity, } \\
\text { contrast, energy, and } \\
\text { entropy }\end{array}$ & $\begin{array}{l}\text { 1) } \mathrm{MDC}+\mathrm{K} \text {-means clustering } \\
\text { 2) } \mathrm{MDC}+\text { proposed GA } \\
\text { 3) } \mathrm{SVM}+\text { proposed GA }\end{array}$ & $\begin{array}{l}\text { Accuracy: } \\
\text { 1) } 86.54 \% \\
\text { 2) } 93.63 \% \\
\text { 3) } 95.71 \%\end{array}$ \\
\hline [11] & $\begin{array}{c}12 \\
\text { species }\end{array}$ & 1,335 & Leaves & $\begin{array}{l}\text { Background } \\
\text { removal using } \\
\text { GAC }\end{array}$ & $\begin{array}{l}\text { Symptom segmentation } \\
\text { - binary mask and } 2 \\
\text { ratios indicating } \\
\text { deviation from green }\end{array}$ & Color histogram & Pairwise classification & $\begin{array}{l}\text { Individual accuracy } \\
\text { range: } 40 \% \text { to } 76 \%\end{array}$ \\
\hline$[12]$ & Apple & 320 & Leaves & $\begin{array}{l}\text { Background } \\
\text { removal, } \\
\text { petiole } \\
\text { removal, and } \\
\text { unwanted noise } \\
\text { removal }\end{array}$ & $\mathrm{K}$-means clustering & Color and texture & $\begin{array}{l}\text { 1) SVM (RBF) } \\
\text { 2) SVM (poly) } \\
\text { 3) ANN }\end{array}$ & $\begin{array}{l}\text { RMSE (testing): } \\
\text { 1) } 0.20 \\
\text { 2) } 0.42 \\
\text { 3) } 0.53 \\
\mathrm{R}^{2} \text { (testing): } \\
\text { 1) } 0.963 \\
\text { 2) } 0.854 \\
\text { 3) } 0.823\end{array}$ \\
\hline [13] & Rice & 500 & Leaves & Not specified & $\begin{array}{l}\text { Fermi energy based } \\
\text { region detection }\end{array}$ & $\begin{array}{l}\text { Color, shape, and } \\
\text { position }\end{array}$ & Rule based classifier & $\begin{array}{c}\text { Classification } \\
\text { accuracy }=92.29 \%\end{array}$ \\
\hline [17] & Wheat & 3,637 & Leaves & $\begin{array}{l}\text { Color } \\
\text { constancy }\end{array}$ & $\begin{array}{l}\text { SLIC and manual } \\
\text { generated masks }\end{array}$ & $\begin{array}{l}\text { Visual features and } \\
\text { use of statistical } \\
\text { inference model }\end{array}$ & Meta classifier & AUC $>0.8$ \\
\hline
\end{tabular}

Based on the stated, it is apparent that many variable factors, e.g., the option of pre-processing techniques, the segmentation methods to be used, the choice of features to be extracted, and the classifier to be used, highly affect the performance of the algorithm. This decision has to be done on a trial and error basis as the performance can vary with the slightest change in one of these factors while using hand crafted features and shallow classifiers. The hand crafted technique is also limited to the number of training samples and the crop and disease range. 


\subsubsection{Classical machine learning with spectral images}

Various imaging techniques that can capture and utilize information beyond a visible range, e.g., hyperspectral, multispectral, thermal, fluorescence imaging, etc., have significantly contributed to the advancement of various plant disease detection aspects [18]. Hyperspectral and multispectral imaging are the most popular imaging technologies that can provide the spatial as well as spectral information of plants which is very useful for evaluation. This study focuses on the work done using hyperspectral and multispectral imaging techniques. The hyperspectral method obtains spectral information from a broader spectral range with narrow wavebands, while the multispectral technique acquires spectral information in comparatively broad wavebands. Abundant spectral details in spectral images help in possible disease detection even before visible disease symptoms appear.

Tian et al. [19] used hyperspectral imaging technology for detecting cucumber downy mildew. They adopted an image fusion technology followed by image enhancement, binarization, corrosion, etc. to get an accuracy of 90\%. Bauriegel et al. [20] and Barbedo et al. [21] studied the detection of fusarium head blight in wheat. Bauriegel et al. [20] analyzed the wheat using hyperspectral images for early detection of fusarium under semi-practical conditions. The authors used principle component analysis (PCA) to identify four spectral ranges that helped in the classification, and reported that stage 75 is the optimal stage for disease detection during the development period scaled by Biologische Bundesanstalt, Bundessortenamt, und Chemische Industrie (BBCH). Spectral angle mapper (SAM) was used to analyze the measure of the disease. However, the study reported SAM to be a time-consuming method so that the study obtained a head blight index for fast identification. Barbedo et al. [21] used hyperspectral imaging for the identification of fusarium head blight in wheat kernels. The output of the algorithm was an index indicating the probability of the kernel being infected. The algorithm achieved a classification accuracy of more than $91 \%$, and was also able to estimate mycotoxin deoxynivalenol concentration in the kernels. The use of a hyperspectral imaging process to detect the most usual defects on orange peels was done in the work of $\mathrm{Li}$ et al. [22]. The study evaluated the hyperspectral images using PCA, band ratio, and a simple thresholding method to get $93.7 \%$ accuracy. However, the study used a small size of only 270 samples for experimentation.

Huang et al. [23] used hyperspectral reflectance to detect the rice leaf folder. A linear regression model was built to study the reflectance captured from rice leaves and the affected canopy at the booting stage. The study established the susceptibility of red, green, and near infrared range to identify rice leaf folder. The model was able to detect a leaf roll rate and infection scale based on spectral indices. It was analyzed using root mean square error (RMSE), and the authors suggested the use of hyperspectral reflectance for detecting rice leaf folder. However, the study was limited to few factors such as crop species, growing stage, species of pests, etc. Zhang et al. [24] studied spectral reflectance from winter wheat to detect powdery mildew. The authors studied hyperspectral reflectance to diagnose powdery mildew of winter wheat. The study analyzed the reflectance from normal and infected leaves in laboratory conditions, and found remarkable spectral changes in the visible and near infrared range. The authors developed multivariate linear regression (MLR) and partial least square regression (PLSR), and also used Fisher's linear discriminant analysis (FLDA) for classifying normal, slight damage, and heavy damage. The work reported that PLSR gave better results compared to MLR for disease severity, and FLDA gave successful results for discrimination analysis of heavily damaged leaves.

Identification of Cercospora leaf spot, leaf rust, and powdery mildew in sugar beet was done in the work of Rumpf et al. [25] and Mahlein et al. [26]. Rumpf et al. [25] used SVM to recognize and classify sugar beet diseases before the symptoms became visible using hyperspectral reflectance. The work classified healthy and diseased leaves with a $97 \%$ accuracy and attained more than $86 \%$ accuracy for multiclass classification. Mahlein et al. [26] built particular disease spectral indices for the identification of sugar beet diseases using hyperspectral signatures. The work used RELIEF-F algorithm to extract the most suitable wavelength and normalized wavelength difference. The method achieved an accuracy of $89 \%$ for healthy sugar beet, 
$92 \%$ for leaf spot, $95 \%$ for powdery mildew, and $87 \%$ for rust. Shi et al. [27] proposed a kernel discriminant algorithm based on spectral vegetation indices to recognize and classify pests and diseases in winter wheat. The work used independent t-tests and correlation analysis to remove redundancy in spectral vegetation indices, and used a Gaussian kernel function for discriminant analysis. The algorithm achieved a leaf level overall accuracy of $82.9 \%$ (slight), 89.2\% (moderate), and $87.9 \%$ (severe) while canopy level accuracy between healthy and damaged leaves was greater than $87 \%$.

Along with hyperspectral imaging, multispectral imaging techniques have also contributed to early plant pathogen detection systems. Cui et al. [28] used multispectral images for detecting soybean rust. The authors used a hue-saturation-intensity (HSI) color model based threshold setting approach for separating the infected region. Two disease diagnostic parameters were extracted for computing rust severity. The study of the center of the leaflet color spread in polar coordinates was also done for automatic rust detection. Aleixos et al. [29] used a multispectral camera to capture visible and near infrared images for citrus defects. The algorithm was implemented using a board based on two digital signal processors (DSPs) to reduce the processing time. The system was also able to classify lemons and mandarins. Dammer et al. [30] compared multispectral and RGB systems to detect winter wheat head blight. The authors found that the multispectral system was better compared to the RGB system, and that in the RGB system, the calibration for the values of R, G, and B in the gray scale channel and modification in thresholds was required for every variety while the multispectral approach required a single calibration before measurements. In the work of Oberti et al. [31], grapevine leaves were imaged using multispectral imaging with five different view angles to investigate the use of sensing computations carried out at an angle for improving the identification sensitivity of powdery mildew. Detection sensitivity was evaluated using an algorithm that combined two spectral indices. The authors reported that the sensitivity increased with view angle in the range of 0 degrees to 75 degrees with the peak at 60 degrees.

Based on the above-mentioned literature, it is clear that multispectral or hyperspectral range images can give an added advantage for pre-symptomatic disease detection. However, due to the limitations such as the cost of these devices being too high, the requirement of specific sensors and calibration, controlled environment usage, selecting appropriate spectral band, etc., their usage is bounded.

Table 2 gives an overview of the work done on plant disease identification using spectral imaging techniques and traditional machine learning algorithms. It compiles the information about the targeted crop and disease, the imaging technology utilized, the index and model adapted, and the evaluation metrics used.

Table 2 Comparative study of classical machine learning algorithm on RGB images

\begin{tabular}{|c|c|c|c|c|c|c|}
\hline Ref. & Crop & Disease & $\begin{array}{l}\text { Imaging } \\
\text { technology }\end{array}$ & Index & Model & Evaluation metric \\
\hline$[20]$ & Wheat & Head blight & $\begin{array}{l}\text { Hyperspectral } \\
\text { imaging }\end{array}$ & $\begin{array}{l}\text { PCA to identify four } \\
\text { spectral ranges }\end{array}$ & $\begin{array}{l}\text { 1) SAM } \\
\text { 2) Head blight index }\end{array}$ & $\begin{array}{l}\text { Accuracy: } \\
\text { 1) } 91 \% \\
\text { 2) } 84 \% \\
\end{array}$ \\
\hline [21] & Wheat & Fusarium head blight & $\begin{array}{l}\text { Hyperspectral } \\
\text { imaging }\end{array}$ & Fusarium index & Morphological operations & Accuracy $>91 \%$ \\
\hline$[22]$ & Orange & 8 common defects & $\begin{array}{l}\text { Hyperspectral } \\
\text { imaging }\end{array}$ & $\begin{array}{l}\text { Band ratio and spectral } \\
\text { features } \\
\end{array}$ & PCA, band ratio, and thresholding & Accuracy $=93.7 \%$ \\
\hline [23] & Rice & $\begin{array}{c}\text { Rice leaf folder } \\
\text { (Cnaphalocrocis } \\
\text { medinalis ) }\end{array}$ & $\begin{array}{l}\text { Hyperspectral } \\
\text { reflectance }\end{array}$ & $\begin{array}{l}\text { Spectral indices: } 38 \text {-leaf } \\
\text { level and 29-canopy level }\end{array}$ & Linear regression & $\begin{array}{l}\text { RMSE: } 0.059 \text {-leaf roll rate and } \\
0.22 \text {-infestation scale }\end{array}$ \\
\hline [24] & $\begin{array}{l}\text { Winter } \\
\text { wheat }\end{array}$ & Powdery mildew & $\begin{array}{l}\text { Hyperspectral } \\
\text { reflectance }\end{array}$ & 32 spectral features & $\begin{array}{l}\text { 1) MLR } \\
\text { 2) PLSR } \\
\text { 3) FLDA }\end{array}$ & $\begin{array}{c}\text { PLSR-(RMSE) }=0.23 \\
\mathrm{R}^{2}=0.8 \\
\text { FLDA-accuracy }=90 \%\end{array}$ \\
\hline [25] & Sugar beet & $\begin{array}{l}\text { Cercospora leaf spot, } \\
\text { leaf rust, and powdery } \\
\text { mildew }\end{array}$ & $\begin{array}{l}\text { Hyperspectral } \\
\text { reflectance }\end{array}$ & $\begin{array}{l}\text { Spectral vegetation } \\
\text { indices }\end{array}$ & $\begin{array}{l}\text { SVM: } \\
\text { 1) Binary classification } \\
\text { 2) Multiclass classification } \\
\end{array}$ & $\begin{array}{l}\text { Accuracy: } \\
\text { 1) } 97 \% \\
\text { 2) } 86 \% \\
\end{array}$ \\
\hline [27] & $\begin{array}{l}\text { Winter } \\
\text { wheat }\end{array}$ & $\begin{array}{l}\text { Yellow rust, aphid, } \\
\text { and powdery mildew }\end{array}$ & $\begin{array}{l}\text { Hyperspectral } \\
\text { reflectance }\end{array}$ & $\begin{array}{l}\text { Spectral vegetation } \\
\text { indices }\end{array}$ & Kernel discriminant analysis & $\begin{array}{c}\text { Leaf level overall accuracy of } \\
\text { occurrence: } 82.9 \% \text { (slight), } 89.2 \% \\
\text { (moderate), and } 87.9 \% \text { (severe) - } \\
\text { accuracy at canopy level }>87 \%\end{array}$ \\
\hline$[28]$ & Soybean & Soybean rust & $\begin{array}{l}\text { Multispectral } \\
\text { imaging }\end{array}$ & $\begin{array}{l}\text { Ratio of infected area } \\
\text { (RIA) and rust color } \\
\text { index (RCI) } \\
\end{array}$ & $\begin{array}{l}\text { Threshold setting and analyzing } \\
\text { the centroid of leaf color } \\
\text { distribution } \\
\end{array}$ & $\begin{array}{l}\text { Increased parameter values with } \\
\text { intensity levels }\end{array}$ \\
\hline$[31]$ & Grapevine & Powdery mildew & $\begin{array}{l}\text { Multispectral } \\
\text { imaging }\end{array}$ & Spectral indices & $\begin{array}{l}\text { Decision region separated by } \\
\text { quadratic function }\end{array}$ & Detection sensitivity $=73 \%$ at $60^{\circ}$ \\
\hline
\end{tabular}




\subsection{Deep learning for plant disease detection}

With recent improvements in areas like artificial intelligence, processor technologies, image processing, and their supporting software, a vital development has been made by deep learning in computer vision technology. It is currently an exceptionally potent research area, and has been applied to many sectors for supervised and unsupervised pattern recognition and classification. In the agriculture sector, it also has been applied to various food production challenges [32]. The potential of deep learning can be leveraged for plant disease diagnosis. One of the famous architectures of deep learning is convolutional neural network $(\mathrm{CNN})$ due to its potential to extract features from the data while training the model. Fig. 3 shows the basic blocks for plant disease detection using deep learning. Deep learning has been proven its potential as feature extractor and classifier in many computer vision tasks thus eliminating the need of feature engineering.

For efficient feature extraction, deep learning architectures need large datasets for their training. However, in the plant disease recognition domain, large and disparate datasets are hardly available. To overcome part of these complexities, the notion of transfer learning is being utilized. Transfer learning is a method of utilizing an existing trained model on a huge dataset for a new related task [33-34].

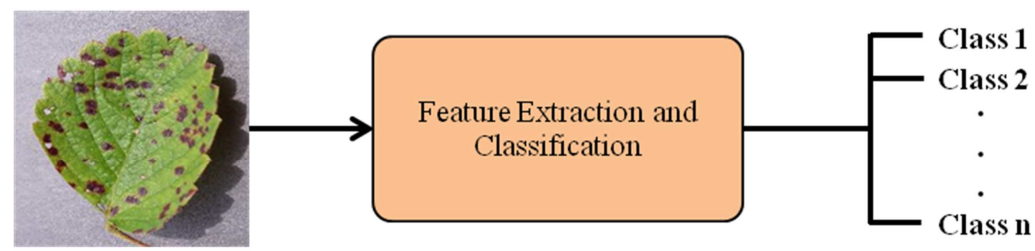

Input

Fig. 3 Classification steps in deep learning

\subsubsection{Deep learning with RGB images}

Numerous plant disease detection techniques based on deep learning algorithms applying to visible band images have been developed in recent years. Jiang et al. [35] used single-shot multibox detector (SSD) for apple disease detection. The authors proposed an improved deep CNN (DCNN) model based upon SSD and an integrated rainbow concatenation method with the improved VGGNet. The model gave 78.80\% mean average precision (mAP) and 23.13 frame per second (FPS) recognition speed. The work also reported that the model was able to recognize more than one disease on the same affected image. Selvaraj et al. [4] proposed using DCNN for the recognition of visible banana infections and pest signs on various parts of banana plants. The authors proposed 6 models (each for different parts of banana plants) and 18 classes, and used transfer learning in the study. ResNet50, InceptionV2, and MobileNetV1models were used for classification. The use of a SSD model with MobileNetV1 was made for fast object detection. These studies demonstrate that the potential of SSD can be utilized for real-time applications including plant disease detection.

Barbedo et al. [36] used a pre-trained network, namely GoogLeNet, for plant disease detection using lesions and spots on leaves. The work involved segmenting original images into individual lesions and spots considering 5 different signs and symptoms such as scattered small, scattered large, isolated, widespread, and powdery. The work reported an improved accuracy using lesions and spots compared to the original images. The authors have made the database public. This study gave an advantage of detecting multiple diseases on the same leaf.

Geetharamani et al. [37] used DCNN to identify 39 classes for 13 different plant leaves taken from plant village dataset. The authors reported an increase in the model performance from $91.43 \%$ to $97.87 \%$ by using data augmentation. They trained their model using various epochs, batch sizes, and dropouts. This work proves that data augmentation helps in increasing recognition performance. 
Coulibaly et al. [33] proposed using transfer learning with visual geometry group-16 (VGG16) to identify mildew disease in millet crops. The model gave a good performance with 95\% accuracy, 90.5\% precision, 94.5\% recall, and 91.75\% F1-score even with a small dataset. Too et al. [38] focused on fine-tuning the well-known CNNs. They evaluated the performance of six different deep architectures by utilizing a dataset for healthy and diseased images of 38 classes. The study proposed that DenseNets gave a better performance concerning the accuracy achieved, parameters required, and time for computation. Mohanty et al. [34] used a dataset of infected and healthy leaves, and trained a DCNN to identify plant varieties and diseases for 14 crop types and 26 diseases. The authors analyzed the performance of two popular architectures AlexNet and GoogleNet for the dataset, and reported that the performance of GoogleNet was better than AlexNet. These studies demonstrated that in cases where there is a lack of large publicly available datasets for plant disease detection, transfer learning can give good performance compared to the models developed from scratch.

Picon et al. [39] extended the work done by Johannes et al. [17], and used DCNN for crop disease classification in the wild. The algorithm used deep residual neural network (ResNet) with advancements in augmentation strategies and tile cropping for disease classification of three wheat diseases for images acquired in real conditions. It gave an improved average balanced accuracy of 0.84 (ResNet) as compared to 0.78 (classical approach), and gave a balanced accuracy of 0.87 for improvements in confidence estimation, superpixel segmentation, and artificial background training. The model also achieved a balanced accuracy of 0.96 on the pilot test. The work demonstrated that the recognition performance for field conditions can be enhanced by adding random images in the background of training images.

Hu et al. [40] proposed the use of a low shot learning technique for the detection of diseases in tea leaves. SVM was used for segmenting the disease spots using color and texture features, and a combination of VGG16 and conditional deep convolutional generative adversarial networks (C-DCGAN) was used to classify tea leaf diseases with an average classification accuracy of $90 \%$.

Ferentinos [41] trained multiple CNN architectures (VGG, Overfeat, AlexNet, GoogLeNet, and AlexNetOWTBn) for plant disease identification using leaf images. The dataset contained 87,848 laboratory and real field condition images. The authors reported that the VGG model gave the highest success rate of $99.53 \%$. However, the authors reported a huge fall in the recognition accuracy when the model trained for laboratory condition images was tested for field condition images and vice versa. This study demonstrated that the model should be trained on large datasets having high variability so that it can generalize effectively on new images, especially the images with field conditions.

Ghazi et al. [42] assessed various factors influencing the performance of DCNN for plant identification using the LifeCLEF 2015 database. They evaluated three deep learning models (GoogLeNet, AlexNet, and VGGNet) by fine-tuning them, and also fused different classifiers to improve the performance. The proposed model gave a validation accuracy of $80 \%$ and 0.752 inverse rank score for the test data. The study also discussed a detailed performance analysis based on essential factors such as batch size, data augmentation, etc., which influence the refining of deep learning models. The study demonstrated that the fusion of different classifiers helps in enhancing classifier performance. It also indicated the impact of data augmentation and hyperparameters on the recognition rate.

Lu et al. [43] proposed a DCNN method for rice disease identification. They used images of leaves and stems for their work. The study utilized a tenfold cross validation strategy and achieved an accuracy of 95.48\%. The authors used stochastic pooling that gave better classification accuracy. Lee et al. [44] proposed useful discriminating features from leaf images to be used for plant identification by CNN. They utilized a deconvolutional network (DN) method to quantify the features that most effectively characterize the leaves. The study reported that the structure of the veins is an essential feature for recognition specifically when the shape feature is not sufficient. The work indicated that a combination of local and global features can raise the identification capability. Table 3 outlines the work done in plant disease recognition with deep learning architectures for processing RGB images. It highlights the crops used, various CNN models, and the evaluation metrics used for the work. 
Table 3 Comparative study of deep learning algorithm on RGB images

\begin{tabular}{|c|c|c|c|c|}
\hline Ref. & Crop & Model/architecture & Dataset/images used & Evaluation metric \\
\hline$[4]$ & Banana & $\begin{array}{c}\text { CNN (ResNet50, InceptionV2, and } \\
\text { MobileNetV1) }\end{array}$ & 30,952 & Accuracy: $70 \%$ to $99 \%$ \\
\hline [33] & Millet & CNN (VGG16) & 124 & $\begin{array}{c}\text { Accuracy }=95 \% \\
\text { Precision }=90.50 \% \\
\text { Recall }=94.50 \% \\
\text { F1-score }=91.75 \%\end{array}$ \\
\hline [34] & $\begin{array}{l}14 \text { plant } \\
\text { species }\end{array}$ & CNN (AlexNet and GoogleNet) & 54,306 & Accuracy $=99.35 \%$ \\
\hline [35] & Apple & CNN (GoogleNet inception module) & 26,377 & $\mathrm{mAP}=78.80 \%$ \\
\hline [36] & 14 crops & GoogLeNet & 46,409 & Accuracy $>75 \%$ \\
\hline [37] & 13 crops & CNN & $\begin{array}{l}\text { Without augmentation }=55,448 \\
\text { With augmentation }=61,486\end{array}$ & Accuracy $=96.46 \%$ \\
\hline [38] & 14 plants & $\begin{array}{c}\text { CNN (VGG16, InceptionV4, } \\
\text { ResNet50/101/152, and DenseNet121) }\end{array}$ & 54,306 & Best accuracy $=99.75 \%$ (for DenseNet) \\
\hline [39] & Wheat & CNN (ResNet) & 8,178 & $\begin{array}{l}\text { Balanced accuracy }>0.96 \text { (improvement } \\
\text { from } 0.78 \text { to } 0.87 \text { for exhaustive testing) }\end{array}$ \\
\hline [40] & Tea & C-DCGAN and VGG16 & 120 & Average accuracy $=90 \%$ \\
\hline [41] & 25 plants & $\begin{array}{l}\text { CNN (AlexNet, AlexNetOWTBn, } \\
\text { GoogLeNet, Overfeat, and VGG) }\end{array}$ & 87,848 & Best accuracy $=99.53 \%($ for VGG) \\
\hline [43] & Rice & DCNN & 500 & Accuracy $=95.48 \%$ \\
\hline
\end{tabular}

Based on the above-mentioned study, it can be stated that data augmentation and transfer learning can be used in cases of unavailability of large datasets for improving the classifier performance. A single CNN can be used for a range of plant diseases across various plant species. The variations in hyperparameters can affect the classification accuracy, and the fusion of classifiers can be used for improving the recognition rate.

\subsubsection{Deep learning with spectral images}

Deep learning is normally applied to RGB images. However, one of the active research areas in plant disease detection is applying deep learning to multispectral and hyperspectral data. Among various imaging techniques, hyperspectral imaging is the most worked technique in the area of deep learning architectures [45]. However, various challenges, such as the size of the data, the increase in computational time due to multidimensional data, the noise in specific bands, the requirement of sufficient training/testing labeled data, and the possibility of high error, need to be addressed while applying hyperspectral data for deep learning.

Nagasubramanian et al. [46] used 3D DCNN to extend its applicability to hyperspectral data. The work integrated the 3D DCNN model with saliency map-based visualization that used the hyperspectral data for the detection of charcoal rot in soybean crops. Using hyperspectral stem images, the model achieved 95.73\% classification accuracy and 0.87 F1-score. The wavelengths in the near infrared region (NIR) were used by the model for classification. The authors reported the limitation of a small dataset (111 hyperspectral stem images with 64-healthy and 47-infected) used in the work. Polder et al. [47] reported a method using modified fully CNN on hyperspectral images for recognition of potato virus Y (PVY) infected potato plant diseases. The study trained the network for real field tests on two rows and validated on two other rows. The precision and recall exceeded 0.78 and 0.88 respectively compared to traditional disease evaluation.

Paoletti et al. [48] and Chen et al. [49] used the Indian pine dataset to get spectral and spatial information of hyperspectral images for classification using CNN architecture. Wang et al. [50] proposed an integrated method using hyperspectral imaging that included segmenting the plant, spectrum classification, and classification of image for early recognition of tomato spotted wilt virus based upon generative adversarial nets (GANs). The method was able to detect infected plants before symptoms appeared, and could categorize the pixels as background pixels, infected plant pixels, and healthy plant pixels without selection of hyperspectral bands. The authors reported $96.25 \%$ accuracy at the plant level. A comprehensive review of deep learning with hyperspectral imaging for multidisciplinary outlook is given in the work of Signoroni et al. [45]. Zhang et al. [51] proposed automated yellow rust disease recognition based upon DCNN and hyperspectral unmanned aerial vehicle (UAV) 
images by utilizing spatial and spectral details. The work proposed Inception-ResNet layers for getting features and reported an overall accuracy of 0.85 . Jin et al. [52] applied deep neural network (DNN) to hyperspectral images for classifying healthy heads and fusarium head blight. The spectra of hyperspectral image pixels were preprocessed to remove the interference occurring due to time intervals and surroundings. The generalization of the model was improved by a hybrid neural network (convolutional layer and bidirectional recurrent layer) that gave an F1-score of 0.75 and an accuracy of 0.743 .

Although a great amount of work utilizing hyperspectral images with deep learning architectures is being done in the area of crop and pest detection, there is still room for improvements in situations like various illumination conditions, images captured with real field background, etc. [53].

\section{Discussion}

Numerous plant disease detection methods are being utilized in order to get early disease detection to control the losses caused by crop pests and diseases. Automated techniques using machine learning algorithms are the most popular techniques for this purpose. These techniques involve analyzing the images captured using various imaging techniques to perform classification. Numerous imaging techniques have been reported in literature, each with its benefits and limitations. Among the numerous available imaging techniques, RGB imaging is the most famous and simple way of capturing images. With the advancements in digital cameras and sensors, this method is being highly accepted. However, a few diseases do not have any corresponding visible signs, or have the signs appearing only when it is too late to take measures. For such cases, normally, the symptoms can only be recognized in the sections of the electromagnetic range beyond the visible scope of humans. A general method in such cases is to utilize a multispectral image, a hyperspectral image, a thermal image, etc. These images have the advantage of pre-symptomatic disease detection. Literature reports that chlorophyll fluorescence and thermography are mainly able to recognize early stress in plants, but they lack detection of specific diseases [18], whereas multispectral and hyperspectral images are found to be superior for specific disease detection. Certainly, a lot of positive research has been carried out using these imaging techniques. Nevertheless, these imaging devices are too costly. They need significant sensors and peculiar devices, and the selection of a proper spectral band is a very important step that can affect the prediction results and the computing time.

While there are a number of imaging technologies that can be used to identify crop pests and diseases, the proper use of algorithms that analyze these images to classify them also plays a major role in the entire process. Although all the early studies in the domain of pest and disease identification were done using classical machine learning algorithms, these algorithms face limitations in terms of the small datasets and the requirement of feature engineering for hand crafted feature extraction leading to limited performance and limited crop and disease scope. Rapid improvements in the computational execution of GPUs, availability of larger datasets, and growth in the assisting software libraries gave rise to an expeditious spurt to experimentations based upon deep learning architectures. The potential of deep learning lies in its ability to handle high dimensional data, its ability to extract relevant features from the data, and its high performance [54-56].

Even if the current work is able to get good classification accuracy for plant disease detection, few challenges still need to be addressed in this area. One of the major challenges for using CNN models in crop disease detection is very few publicly available datasets. Plant village dataset [7] and plant disease symptoms (PDDB) dataset [11, 36, 57] are the only two known publicly available datasets. It has been noted that researchers capture images for creating their datasets without making them publicly available. Availability of a large number of datasets for use will allow comparing the results with the work of other researchers and allow testing the proposed method with the images not included in their database. To overcome the problem of sufficient dataset, techniques like data augmentation, GAN [50], and transfer learning techniques have been confirmed to enhance the model performance. Barbedo et al. [36] suggested the use of individual lesions and spots as another way of augmenting data to get a large dataset. Another important aspect to be considered is that most of the work done is on the images 
captured in controlled surroundings. The images captured in the real field environment will affect the performance of the model, and hence the focus should be given on analyzing the images captured in natural conditions, with special attention given to complex background removal. Also, the CNN architectures built from scratch need large storage requirements and high computational time. Having a compact deep learning model in a mobile or embedded system is an essential need. Models like MobileNet, SqueezeNet, and ShuffleNet [58] that need less storage and training time are being used for smartphone deployment for such applications. A compact CNN architecture would be beneficial for mobile applications for real-time plant disease detection and would be beneficial to farmers.

\section{Conclusions}

This study presents an extensive review of the current work done in plant disease detection using various imaging techniques in combination with conventional machine learning and deep learning architectures. The study reports that in recent years, CNN models have superseded traditional machine learning models for crop disease detection as they give significantly higher accuracy levels and a broad range of detection in terms of plant species and diseases. However, they need large databases for training the model to achieve high accuracy and precision. The study also discusses the lack of publicly available datasets, the lack of images captured in natural conditions, and the limitations resulting from these.

The use of various imaging techniques, for getting the maximum possible information to achieve early disease detection, is also discussed in the study. Among the numerous imaging techniques, RGB imaging is the most popular method. However, it lacks pre-symptomatic disease detection. The use of hyperspectral and multispectral images can be used to achieve this pre-symptomatic disease detection. However, there are issues to be addressed, such as challenges of processing high dimensional data, huge amounts of computational time required, noises in the spectral band, perfect band selections, etc.

With continuous advancements in technologies of imaging sensors, GPUs, and computer vision, it can be expected that in the near future, smartphones with built-in sophisticated sensors and compact deep learning architecture will be utilized for the real-time, rapid, accurate, and early disease detection, for a huge variety of plants and diseases to control economical and agricultural losses.

\section{Conflicts of Interest}

The authors declare no conflict of interest.

\section{References}

[1] “Agriculture in India: Information about Indian Agriculture \& Its Importance," https://www.ibef.org/industry/agriculture-india.aspx, July 28, 2021.

[2] J. G. A. Barbedo, "Digital Image Processing Techniques for Detecting, Quantifying and Classifying Plant Diseases," SpringerPlus, vol. 2, no. 1, 660, December 2013.

[3] M. Sharif, M. A. Khan, Z. Iqbal, M. F. Azam, M. I. U. Lali, and M. Y. Javed, "Detection and Classification of Citrus Diseases in Agriculture Based on Optimized Weighted Segmentation and Feature Selection," Computers and Electronics in Agriculture, vol. 150, pp. 220-234, July 2018.

[4] M. G. Selvaraj, A.Vergara, H. Ruiz, N. Safari, S. Elayabalan, W. Ocimati, et al., "AI-Powered Banana Diseases and Pest Detection," Plant Methods, vol. 15, no. 1, 92, December 2019.

[5] H. Ali, M. I. Lali, M. Z. Nawaz, M. Sharif, and B. A. Saleem, "Symptom Based Automated Detection of Citrus Diseases Using Color Histogram and Textural Descriptors," Computers and Electronics in Agriculture, vol. 138, pp. 92-104, May 2017.

[6] M. Islam, A. Dinh, K. Wahid, and P. Bhowmik, "Detection of Potato Diseases Using Image Segmentation and Multiclass Support Vector Machine," IEEE 30th Canadian Conference on Electrical and Computer Engineering, April 2017, pp. 1-4.

[7] D. Hughes and M. Salathé, "An Open Access Repository of Images on Plant Health to Enable the Development of Mobile Disease Diagnostics," https://arxiv.org/ftp/arxiv/papers/1511/1511.08060.pdf, April 12, 2016.

[8] M. Zhang and Q. Meng, "Automatic Citrus Canker Detection from Leaf Images Captured in Field," Pattern Recognition Letters, vol. 32, no. 15, pp. 2036-2046, November 2011. 
[9] A. E. Hassanien, T. Gaber, U. Mokhtar, and H. Hefny, “An Improved Moth Flame Optimization Algorithm Based on Rough Sets for Tomato Diseases Detection," Computers and Electronics in Agriculture, vol. 136, pp. 86-96, April 2017.

[10] V. Singh and A. K. Misra, "Detection of Plant Leaf Diseases Using Image Segmentation and Soft Computing Techniques," Information Processing in Agriculture, vol. 4, no. 1, pp. 41-49, March 2017.

[11] J. G. A. Barbedo, L. V. Koenigkan, and T. T. Santos, "Identifying Multiple Plant Diseases Using Digital Image Processing," Biosystems Engineering, vol. 147, pp. 104-116, July 2016.

[12] E. Omrani, B. Khoshnevisan, S. Shamshirband, H. Saboohi, N. B. Anuar, and M. H. N. M. Nasir, "Potential of Radial Basis Function-Based Support Vector Regression for Apple Disease Detection,” Measurement, vol. 55, pp. 512-519, September 2014.

[13] S. Phadikar, J. Sil, and A. K. Das, "Rice Diseases Classification Using Feature Selection and Rule Generation Techniques," Computers and Electronics in Agriculture, vol. 90, pp. 76-85, January 2013.

[14] J. G. A. Barbedo, “An Automatic Method to Detect and Measure Leaf Disease Symptoms Using Digital Image Processing," Plant Disease, vol. 98, no. 12, pp. 1709-1716, December 2014.

[15] A. Camargo and J. S. Smith, “An Image-Processing Based Algorithm to Automatically Identify Plant Disease Visual Symptoms," Biosystems Engineering, vol. 102, no. 1, pp. 9-21, January 2009.

[16] A. Camargo and J. S. Smith, "Image Pattern Classification for the Identification of Disease Causing Agents in Plants," Computers and Electronics in Agriculture, vol. 66, no. 2, pp. 121-125, May 2009.

[17] A. Johannes, A. Picon, A. Alvarez-Gila, J. Echazarra, S. Rodriguez-Vaamonde, A. D. Navajas, et al., "Automatic Plant Disease Diagnosis Using Mobile Capture Devices, Applied on a Wheat Use Case," Computers and Electronics in Agriculture, vol. 138, pp. 200-209, June 2017.

[18] A. K. Mahlein, "Plant Disease Detection by Imaging Sensors-Parallels and Specific Demands for Precision Agriculture and Plant Phenotyping," Plant Disease, vol. 100, no. 2, pp. 241-251, February 2016.

[19] Y. Tian and L. Zhang, "Study on the Methods of Detecting Cucumber Downy Mildew Using Hyperspectral Imaging Technology,” Physics Procedia, vol. 33, pp. 743-750, 2012.

[20] E. Bauriegel, A. Giebel, M. Geyer, U. Schmidt, and W. B. Herppich, "Early Detection of Fusarium Infection in Wheat Using Hyper-Spectral Imaging," Computers and Electronics in Agriculture, vol. 75, no. 2, pp. 304-312, February 2011.

[21] J. G. Barbedo, C. S. Tibola, and J. M. Fernandes, "Detecting Fusarium Head Blight in Wheat Kernels Using Hyperspectral Imaging,” Biosystems Engineering, vol. 131, pp. 65-76, March 2015.

[22] J. Li, X. Rao, and Y. Ying, "Detection of Common Defects on Oranges Using Hyperspectral Reflectance Imaging," Computers and Electronics in Agriculture, vol. 78, no. 1, pp. 38-48, August 2011.

[23] J. Huang, H. Liao, Y. Zhu, J. Sun, Q. Sun, and X. Liu, "Hyperspectral Detection of Rice Damaged by Rice Leaf Folder (Cnaphalocrocis Medinalis)," Computers and Electronics in Agriculture, vol. 82, pp. 100-107, March 2012.

[24] J. C. Zhang, R. L. Pu, J. H. Wang, W. J. Huang, L. Yuan, and J. H. Luo, "Detecting Powdery Mildew of Winter Wheat Using Leaf Level Hyperspectral Measurements," Computers and Electronics in Agriculture, vol. 85, pp. 13-23, July 2012.

[25] T. Rumpf, A. K. Mahlein, U. Steiner, E. C. Oerke, H. W. Dehne, and L. Plümer, "Early Detection and Classification of Plant Diseases with Support Vector Machines Based on Hyperspectral Reflectance," Computers and Electronics in Agriculture, vol. 74, no. 1, pp. 91-99, October 2010.

[26] A. K. Mahlein, T. Rumpf, P. Welke, H. W. Dehne, L. Plümer, U. Steiner, et al., "Development of Spectral Indices for Detecting and Identifying Plant Diseases,” Remote Sensing of Environment, vol. 128, pp. 21-30, January 2013.

[27] Y. Shi, W. Huang, J. Luo, L. Huang, and X. Zhou, "Detection and Discrimination of Pests and Diseases in Winter Wheat Based on Spectral Indices and Kernel Discriminant Analysis," Computers and Electronics in Agriculture, vol. 141, pp. 171-180, September 2017.

[28] D. Cui, Q. Zhang, M. Li, G. L. Hartman, and Y. Zhao, "Image Processing Methods for Quantitatively Detecting Soybean Rust from Multispectral Images,” Biosystems Engineering, vol. 107, no. 3, pp. 186-193, November 2010.

[29] N. Aleixos, J. Blasco, F. Navarrón, and E. Moltó, "Multispectral Inspection of Citrus in Real-Time Using Machine Vision and Digital Signal Processors," Computers and Electronics in Agriculture, vol. 33, no. 2, pp. 121-137, February 2002.

[30] K. H. Dammer, B. Möller, B. Rodemann, and D. Heppner, "Detection of Head Blight (Fusarium Ssp.) in Winter Wheat by Color and Multispectral Image Analyses," Crop Protection, vol. 30, no. 4, pp. 420-428, April 2011.

[31] R. Oberti, M. Marchi, P. Tirelli, A. Calcante, M. Iriti, and A. N. Borghese, "Automatic Detection of Powdery Mildew on Grapevine Leaves by Image Analysis: Optimal View-Angle Range to Increase the Sensitivity,” Computers and Electronics in Agriculture, vol. 104, pp. 1-8, June 2014.

[32] A. Kamilaris and F. X. Prenafeta-Boldú, "Deep Learning in Agriculture: A Survey," Computers and Electronics in Agriculture, vol. 147, pp. 70-90, April 2018.

[33] S. Coulibaly, B. Kamsu-Foguem, D. Kamissoko, and D. Traore, "Deep Neural Networks with Transfer Learning in Millet Crop Images," Computers in Industry, vol. 108, pp. 115-120, June 2019.

[34] S. P. Mohanty, D. P. Hughes, and M. Salathé, "Using Deep Learning for Image-Based Plant Disease Detection,” Frontiers in Plant Science, vol. 7, 1419, September 2016. 
[35] P. Jiang, Y. Chen, B. Liu, D. He, and C. Liang, "Real-Time Detection of Apple Leaf Diseases Using Deep Learning Approach Based on Improved Convolutional Neural Networks," IEEE Access, vol. 7, pp. 59069-59080, 2019.

[36] J. G. A. Barbedo, "Plant Disease Identification from Individual Lesions and Spots Using Deep Learning," Biosystems Engineering, vol. 180, pp. 96-107, April 2019.

[37] G. Geetharamani and A. Pandian, "Identification of Plant Leaf Diseases Using a Nine-Layer Deep Convolutional Neural Network," Computers and Electrical Engineering, vol. 76, pp. 323-338, June 2019.

[38] E. C. Too, L. Yujian, S. Njuki, and L. Yingchun, “A Comparative Study of Fine-Tuning Deep Learning Models for Plant Disease Identification,” Computers and Electronics in Agriculture, vol. 161, pp. 272-279, June 2019.

[39] A. Picon, A. Alvarez-Gila, M. Seitz, A. Ortiz-Barredo, J. Echazarra, and A. Johannes, "Deep Convolutional Neural Networks for Mobile Capture Device-Based Crop Disease Classification in the Wild," Computers and Electronics in Agriculture, vol. 161, pp. 280-290, June 2019.

[40] G. Hu, H. Wu, Y. Zhang, and M. Wan, “A Low Shot Learning Method for Tea Leaf's Disease Identification,” Computers and Electronics in Agriculture, vol. 163, 104852, August 2019.

[41] K. P. Ferentinos, "Deep Learning Models for Plant Disease Detection and Diagnosis," Computers and Electronics in Agriculture, vol. 145, pp. 311-318, February 2018.

[42] M. M. Ghazi, B. Yanikoglu, and E. Aptoula, "Plant Identification Using Deep Neural Networks via Optimization of Transfer Learning Parameters," Neurocomputing, vol. 235, pp. 228-235, April 2017.

[43] Y. Lu, S. Yi, N. Zeng, Y. Liu, and Y. Zhang, "Identification of Rice Diseases Using Deep Convolutional Neural Networks," Neurocomputing, vol. 267, pp. 378-384, December 2017.

[44] S. H. Lee, C. S. Chan, S. J. Mayo, and P. Remagnino, "How Deep Learning Extracts and Learns Leaf Features for Plant Classification,” Pattern Recognition, vol. 71, pp. 1-13, November 2017.

[45] A. Signoroni, M. Savardi, A. Baronio, and S. Benini, "Deep Learning Meets Hyperspectral Image Analysis: A Multidisciplinary Review,” Journal of Imaging, vol. 5, no. 5, 52, May 2019.

[46] K. Nagasubramanian, S. Jones, A. K. Singh, S. Sarkar, A. Singh, and B. Ganapathysubramanian, "Plant Disease Identification Using Explainable 3D Deep Learning on Hyperspectral Images," Plant Methods, vol. 15, no. 1, 98, December 2019.

[47] G. Polder, P. M. Blok, H. A. de Villiers, J. M. van der Wolf, and J. Kamp, "Potato Virus Y Detection in Seed Potatoes Using Deep Learning on Hyperspectral Images," Frontiers in Plant Science, vol. 10, 209, March 2019.

[48] M. E. Paoletti, J. M. Haut, J. Plaza, and A. Plaza, “A New Deep Convolutional Neural Network for Fast Hyperspectral Image Classification,” ISPRS Journal of Photogrammetry and Remote Sensing, vol. 145, pp. 120-147, November 2018.

[49] Y. Chen, H. Jiang, C. Li, X. Jia, and P. Ghamisi, "Deep Feature Extraction and Classification of Hyperspectral Images Based on Convolutional Neural Networks," IEEE Transactions on Geoscience and Remote Sensing, vol. 54, no. 10, pp. 6232-6251, October 2016.

[50] D. Wang, R. Vinson, M. Holmes, G. Seibel, A. Bechar, S. Nof, et al., "Early Detection of Tomato Spotted Wilt Virus by Hyperspectral Imaging and Outlier Removal Auxiliary Classifier Generative Adversarial Nets (OR-AC-GAN)," Scientific Reports, vol. 9, 4377, 2019.

[51] X. Zhang, L. Han, Y. Dong, Y. Shi, W. Huang, L. Han, et al., “A Deep Learning-Based Approach for Automated Yellow Rust Disease Detection from High-Resolution Hyperspectral UAV Images," Remote Sensing, vol. 11, no. 13, 1554, July 2019.

[52] X. Jin, L. Jie, S. Wang, H. J. Qi, and S. W. Li, "Classifying Wheat Hyperspectral Pixels of Healthy Heads and Fusarium Head Blight Disease Using A Deep Neural Network in the Wild Field,” Remote Sensing, vol. 10, no. 3, 395, March 2018.

[53] K. Golhani, S. K. Balasundram, G. Vadamalai, and B. Pradhan, "A Review of Neural Networks in Plant Disease Detection Using Hyperspectral Data,” Information Processing in Agriculture, vol. 5, no. 3, pp. 354-371, September 2018.

[54] R. Sujatha, J. M. Chatterjee, N. Z. Jhanjhi, and S. N. Brohi, "Performance of Deep Learning vs Machine Learning in Plant Leaf Disease Detection," Microprocessors and Microsystems, vol. 80, 103615, February 2021.

[55] L. C. Ngugi, M. Abelwahab, and M. Abo-Zahhad, "Recent Advances in Image Processing Techniques for Automated Leaf Pest and Disease Recognition-A Review," Information Processing in Agriculture, vol. 8, no. 1, pp. 27-51, March 2021.

[56] R. Bhagwat and Y. Dandawate, "A Framework for Crop Disease Detection Using Feature Fusion Method," International Journal of Engineering and Technology Innovation, vol. 11, no. 3, pp. 216-228, June 2021.

[57] J. G. A. Barbedo, L. V. Koenigkan, B. A. Halfeld-Vieira, R. V. Costa, K. L. Nechet, C. V. Godoy, et al., "Annotated Plant Pathology Databases for Image-Based Detection and Recognition of Diseases," IEEE Latin America Transactions, vol. 16, no. 6, pp. 1749-1757, June 2018.

[58] X. Zhang, X. Zhou, M. Lin, and J. Sun, "Shufflenet: An Extremely Efficient Convolutional Neural Network for Mobile Devices," IEEE Conference on Computer Vision and Pattern Recognition, June 2018, pp. 6848-6856.

Copyright $@$ by the authors. Licensee TAETI, Taiwan. This article is an open access article distributed under the terms and conditions of the Creative Commons Attribution (CC BY-NC) license (https://creativecommons.org/licenses/by-nc/4.0/). 